\title{
Vouloir pour la Nation. Le concept de représentation dans la théorie de l'État
}

Jean Bart

\section{(2) OpenEdition}

1 Journals

Édition électronique

URL : https://journals.openedition.org/ahrf/2227

DOI : 10.4000/ahrf.2227

ISSN : 1952-403X

Éditeur :

Armand Colin, Société des études robespierristes

Édition imprimée

Date de publication : 1 septembre 2005

Pagination : 195-197

ISSN : 0003-4436

\section{Référence électronique}

Jean Bart, « Vouloir pour la Nation. Le concept de représentation dans la théorie de l'État », Annales historiques de la Révolution française [En ligne], 341 | juillet-septembre 2005, mis en ligne le 27 avril 2006, consulté le 24 avril 2022. URL : http://journals.openedition.org/ahrf/2227 ; DOI : https://doi.org/ 10.4000/ahrf.2227

Ce document a été généré automatiquement le 24 avril 2022.

Tous droits réservés 


\section{Vouloir pour la Nation. Le concept de représentation dans la théorie de l'État}

Jean Bart

\section{RÉFÉRENCE}

Pierre Brunet, Vouloir pour la Nation. Le concept de représentation dans la théorie de l'État, Rouen - Paris - Bruxelles, Publications de l'Université de Rouen - L.G.D.J. - Bruylant, 2004, 396 p., ISBN : 2-275-02518-9, 25 e.

1 Les lecteurs de notre revue ne manqueront pas d'être déconcertés par ce livre. Alors que, au sein de l'histoire politique en renouveau, l'étude des phénomènes électoraux suit des voies jusqu'alors délaissées et riches en découvertes - que l'on songe, entre autres, au déjà célèbre Guide pour la recherche. Voter, élire pendant la Révolution française, 1789-1799, Paris, CTHS, 1999, concluant un séminaire particulièrement fécond , ou au livre de Philippe Tanchoux, Les procédures électorales en France de la fin de l'Ancien Régime à la première guerre mondiale, Paris, CTHS, 2004 -, Pierre Brunet tourne résolument le dos à l'histoire. Pour cet analyste du droit positif, la science juridique n'a eu que trop tendance à regarder en arrière; attitude dangereuse à ses yeux, car "prétendre prouver la pertinence de concepts contemporains, à l'aide des catégories du passé c'est se condamner à la circularité et courir le risque de confondre la description scientifique avec sa propre idéologie [...]. L'utilisation de l'histoire à des fins scientifiques est donc le premier danger auquel on doit faire face ». La connaissance du fait électoral est donc complètement étrangère à l'auteur. Ce qui l'intéresse, c'est, selon le sous-titre tout à fait adéquat du livre, saisir le concept de représentation dans la théorie de l'État. Et si de nombreux textes issus de discours de l'époque révolutionnaire sont utilisés à cette fin, et cités, en vertu d'un "anachronisme des concepts " non seulement conscient mais voulu, c'est "pour rendre intelligible, non pas des faits, mais des discours ou représentations de ces faits. Il ne s'agit pas de "retrouver" le présent dans le passé ", 
mais de "comprendre, décrire, expliquer ce qui a été fait ou dit à l'aide d'outils contemporains dont on sait que les acteurs ne les possédaient pas ». Moyennant quoi, est affirmée l'incapacité des historiens à suivre une telle méthode que seuls les juristes pourraient mettre en action, n'en déplaise - mais c'est nous qui précisons - aux tenants naïs de l'interdisciplinarité. La perspective historique étant ainsi évacuée, Pierre Brunet se propose de démontrer que, loin d'être une théorie, issue de la pensée des Lumières - dont toute influence est niée -, sur laquelle reposeraient les bases du gouvernement démocratique, la représentation n'est qu'une justification - maître mot de la thèse - du pouvoir, fabriquée après coup pour faire admettre le système établi.

2 C'est donc à un voyage au pays de la " théorie pure du droit » que nous convie l'auteur, dans un cadre chronologique allant de la fin de l'Ancien Régime à la Ve République, les trois quarts de l'ouvrage étant nourris de textes de la période révolutionnaire. Mais, bien sûr, le plan n'est pas - ne peut pas - être chronologique. Selon une construction bien balancée dont les juristes ont le goût, l'ouvrage est divisé en deux parties : I. La représentation de l'unité, II. L'unité de la représentation.

Il faut donc d'abord rechercher comment « faire de toutes les parties un seul corps ». À cette fin, sont revisitées les notions de base de l'analyse classique que sont la Nation, la souveraineté et la représentation, à la lumière tant de l'opposition devenue traditionnelle entre la souveraineté nationale et la souveraineté populaire - et de leur supposée incompatibilité -, que des thèses classiques de la théorie générale de l'État, d'Hobbes à Carré de Malberg et Kelsen, avec une analyse très fouillée du concept d'organe. Dès lors la représentation apparaît comme une construction rhétorique " permettant à chacun de jouir du sentiment d'indépendance pendant que certains sont chargés de s'occuper du collectif ». Et P.B. de chercher à démontrer, en inversant la conception ordinaire, que « la Nation n'est pas une personne distincte de ceux qui la constituent et ayant, pour agir, besoin de représentants mais cette personne formée par l'action des représentants ». Pour bâtir leur "justification», les hommes de la Révolution n'ont eu qu'à puiser dans le vocabulaire et les idées des parlements en lutte contre l'absolutisme monarchique, qui voulaient apparaitre eux-mêmes comme les représentants de la Nation et prendre part à l'exercice du pouvoir législatif. À leur profit, les députés du Tiers ont réussi à faire admettre que «le corps politique n'est désormais plus contenu dans le roi mais dans l'Assemblée laquelle, comme le roi, prend alternativement les traits $\mathrm{du}$ corps tout entier ou de sa tête", et qui, tout naturellement, jouit du pouvoir de faire la loi. Jouant un peu sur les mots, l'auteur va jusqu'à affirmer que le célèbre vote du 17 juin 1789 n'a pas opéré, comme on le dit généralement, une substitution de la Nation au roi, mais une simple «substitution de justification ».

4 Cependant, sur le plan logique, cette première justification ne suffit pas. Reste encore à résoudre deux paradoxes. D'abord, si ceux qui parlent au nom de la Nation « doivent être libres, [...] une fois libres, comment garantir qu'ils continueront de se considérer comme partie de l'unité ?». Tel est le « dilemme de l'organe » : si l'organe (l'Assemblée nationale) impose sa volonté, "il risque de primer l'ensemble dont il n'est que l'organe ». D'où l'interdiction des mandats impératifs, Sieyès faisant admettre dès l'été 1789, que la volonté de la Nation exprimée par les représentants ne peut être identifiée avec celle de chacun d'eux. Et même en l'an I, Girondins comme Montagnards admettront que la liberté des représentants ne porte pas atteinte à la souveraineté du peuple, préoccupation qui, en l'an III, n'est plus à l'ordre du jour. Le second paradoxe 
réside dans la décision «de faire une unité par la division ». Autrement dit, puisque tous ceux qui agissent pour appliquer la volonté de la Nation sont élus dans les différents rouages de l'administration locale, tous les élus sont-ils considérés comme des représentants - libres par essence-, ou sont-ils soumis à une autorité estimée supérieure? Aussi, après avoir démontré la représentation de l'unité, était-il nécessaire, pour mieux justifier cette soumission, d'affirmer l'unité de la représentation. Après avoir fait de l'élection le ciment de l'unité du territoire et à la suite de quelques hésitations entre 1789 et 1791, le titre de représentant fut réservé définitivement à ceux qui reçoivent mission de poser les normes applicables à tous, les élus chargés d'appliquer ces normes n'étant que des administrateurs, ou "fonctionnaires", au sens que ce mot revêtait alors. La démonstration de cette construction théorique, reposant sur des textes de l'époque révolutionnaire et de leur évolution, se poursuit par l'analyse du concept de représentation, d'abord dans les chartes de la monarchie parlementaire, puis au regard de l'évolution des pouvoirs sous la Ve République. Cependant, à aucun de ces moments ne peut être saisie une «théorie dogmatique de la représentation d'où l'on aurait déduit des règles constitutionnelles »; la représentation n'est ni un mythe, ni une fiction, ce n'est toujours... qu'une justification.

5 En définitive, malgré la nécessité, reconnue çà et là par l'auteur, de suivre parfois les méandres de l'histoire, le concept de représentation est analysé in vitro. L'essai est brillant. Sera-t-il de quelque secours aux chercheurs de terrain qui renouvellent aujourd'hui l'histoire des élections? On peut en douter. Il est vrai que tel n'était pas la préoccupation de l'auteur. 